\title{
Analysis of the Factors Affecting the Calibration of an Earth Pressure Cell and Improvement Measures
}

\author{
Fengjun Liu' ${ }^{1, a}$, Shijie Feng ${ }^{1, b}$ \\ ${ }^{1}$ School of Civil Engineering, Henan University of Science \& Technology, \\ 263 Kaiyuan Avenue, Luoyang, Henan, China \\ alfjhaust@163.com, byouian2009@126.com
}

\begin{abstract}
Keywords: Earth pressure cell, Calibration, Factor, Improvement measure Abstract. Earth pressure is usually measured by the earth pressure cell. Calibration parameters from the manufacturer is often obtained conducting calibration in the gas or liquid systematically with a set of pressure, which can not be used in soil test directly. Therefore, in order to find out a well-matched curve to target work environment, calibration of earth pressure cells should be done in the simulation or target environment before their use. In this paper, based on the research outcome so far, factors affecting the calibration are analyzed, some preventive and improvement measures are put forward, which is expected to benefit the choice and the calibration of earth pressure cells.
\end{abstract}

\section{Introduction}

The accurate measurement of earth pressure plays a key role in experimental and theoretical study on soil mechanics and is a crucial element in the geotechnical testing technology. Generally speaking, earth pressure is determined by the earth pressure cell. Manufacturers of earth pressure cells mostly adopt pneumatic or hydraulic calibration, which is not consistent with its real working environment, and this results in distortion of measured data using earth pressure cells. Chunlin $\mathrm{Li} \mathrm{[1]} \mathrm{and} \mathrm{Chunhong}$ Chen [2] showed that there is a significant difference between the results of oil pressure calibration and sand calibration, and the maximum difference is up to 29.3-36.7\%. Shuyan Zheng [3] conducted sand calibration, hydraulic calibration and finite element simulation of earth pressure cells, and the results showed that sand calibration value is closer to numerical calculation value, and the value of hydraulic calibration value is up to $30.3 \%$ higher than that of sand calibration. Therefore, an earth pressure cell should have a calibration in the simulation or real target environment to obtain a calibration curve for the target environment before its use. Earth pressure calibration value is affected by many factors, Sijiang Wei[4], Bin Zhang[5] and others did some research and analysis, but the research or analysis is limited to certain aspects, and lacks of systematization.

Based on the research of scholars at home and abroad, this paper makes a systematic analysis of the factors affecting the measured value by the earth pressure cell and offers some improvement measures, in order to provide some help for the selection and calibration of earth pressure cells.

\section{Factors Affecting the Calibration of Earth Pressure Cell}

The error between the measured value of the cell pressure cell and the original stress value of the point is called matching error. In this regard, domestic and foreign scholars have done a lot of research in both theory and experiment, and agreed that the thickness-to-diameter ratio $\mathrm{H} / \mathrm{D}$ of the earth pressure cell and the modulus ratio Eg / Es between the earth pressure cell and the measured medium are the core factors. However, the measured value of an earth pressure cellis comprehensively affected by lots of factors.

\section{Properties of Earth Pressure Cell}

Thickness-to-diameter ratio H / D. Theoretical formulae derived by Д.С.Бараноь, Р.А.Аббот, Askergcard V. and Hui Zeng show that the thickness-to-diameter ratio is a core factor affecting measured values by earth pressure cells[6]. Lixiang Zhang[7] using the finite element method to establish a model of interaction between the earth pressure cell and pan soil, the results showed that the absolute value of the matching error increases linearly with the increase of the value of $\mathrm{D} / \mathrm{H}$. The 
smaller the value of $\mathrm{D} / \mathrm{H}$, the closer to the actual stress for the measured results, and generally, it is recommended that the value of $\mathrm{D} / \mathrm{H}$ should not be larger than 0.1-0.2.

Deflection. The deflection of the earth pressure cell has important influence on the measured value. In 1947, Taylor D. W. put forward the reasonable deflection of the pressure plate of the earth pressure cell is $\delta=\mathrm{D} / 5000$; in 1956, using loop instrument to do calibration in sand and round gravel, Kallstenius T. thought that $\delta=\mathrm{D} / 10000$ would be better; and, after calibration in sand mulling and dinas, Nunohara Matsudaira et al. thought arch effect could be not taken into consideration when $\delta=\mathrm{D} / 10000[8]$.

Hysteresis. The hysteresis of the earth pressure cell is an important index to reflect its process, which has a great relationship with the stability, uniformity, and metallurgical structure after heat treatment of the material itself, and the value of which should be no more than $3 \%$. The hysteresis can be reduced by improving the elastic limit or through itself compensation in order that the influence of the hysteresis is reduced to the lowest level. Yuanming Zuo[9] test showed that the hysteresis of of the earth pressure cell with thin film is large, on the contrary, with thick film is small.

Sensitivity. The sensitivity too low, the earth pressure cell is slow to react, and counterproductive; The sensitivity too high, it requires a high level of membrane processing technology and it's difficult to guarantee the stability of the measured value. YongLi Xie[10] thought that if earth pressure $10 \mathrm{kN} / \mathrm{m} 2$ the output of micro strain is more than $100 \mathrm{u} \varepsilon$, it can meet test requirements even considering of "zero drift".

Zero drift. Zero drift is inevitable for electronic components. The experiment found out that under normal circumstances, zero drift of full load is with repeatability, and $4 \%$ is recommended as a limit indicator[7].

\section{Properties of Medium}

Modulus of elasticity. Soil's modulus of elasticity is a variable, while modulus elasticity of the earth pressure cell is is relatively constant in its effective range, which makes it difficult to maintain the consistency of the stiffness ratio between the earth pressure cell and the measured soil. The earth pressure cell's embedding destroys the transmission path of the original force chain of the measured medium, and the earth pressure generates stress redistribution in a certain range around it. The stress the earth pressure cell bears is not equal to the original stress, that is, the "soil arch" effect. If the rigidity of the earth pressure cell is larger than that of the measured medium, the measured result is larger than the real, and vice versa. Joseph et al. [11] showed that stiffness ratio between the earth pressure cell and the soil is greater than 20, the measured value will be $10 \%$ larger than the real.

Grain size. Grain size has a significant influence on the measured value, and many scholars have carried out research on it. Diping Hang[12] found out the difference of K value measured is within $10 \%$ under the same condition through the comparison of 3 groups of curves from the calibration in loess and sand. Yongxing Guo et al. [13] conducted pressure tests of 3 kinds of sand medium similar to the working environment, and the results showed that the inhomogeneity of the medium particles has a great impact on the measurement results. Sijiang Wei et al. [4] did the calibration of miniature earth pressure cells in granular materials (fine sand, medium sand, coarse sand), powders (800 mesh light calcium carbonate, 120 mesh gypsum) and similar materials (condensed from a certain proportion of mixture of fine sand, gypsum and calcium carbonate and water), and compared with the given $\mathrm{K}$ by the manufacturer, the resluts showed there is $4.79-51.77 \%$ difference between them and

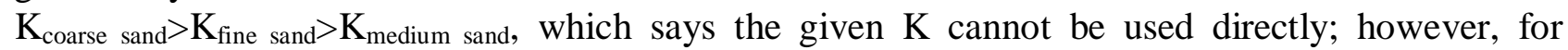
compacted powders and similar materials, the measured $\mathrm{K}$ is slightly greater than the given $\mathrm{K}$ and the given $\mathrm{K}$ can be used directly. Bin Zhang[5] considered to reduce the error caused by stress concentration, the diameter of the effective pressure-bearing part of the earth pressure cell deformation film must be more than 50 times the maximum diameter of the measured medium. Shengli Zhang [6] calibrating GYI-11 sensor with six kinds of fractions, there exists nonlinear relationship between the output curve slope of earth pressure cells and medium size, and medium particle diameter should be less than 1/4-1/ 3 that of the earth pressure cell to get calibration curves with good regression. 
Degree of density. In order to ensure the accuracy of measurement, calibiration tests under the different degree of density should be carried out. Sijiang Wei et al. [4] showed that the K difference of uncompacted granular materials is smaller than that of the compacted homogeneous. Xianshan Liu et al.[14] analyzed two samples with different porosity using a particle flow numerical model of sand and earth pressure cell, and thought particles with larger porosity has a compaction process under the load and requires larger load to reach the same value. Yabing Qiu et al.[15] did calibration in the stone grading-scale gravel, and the results showed that with the increase of dry density, earth pressure cell readings tend to vary linearly.

\section{Environmental Effect}

Temperature. Temperature is the most prominent among the environment fators 。 Daigle L.[16] showed that test results of earth pressure cells have close relationship with temperature change through four years of trials, and found out that the larger the load applied to the earth pressure cells and the smaller the range, the larger the influence of ambient temperature, especially for the shallow-buried earth pressure cells. Fengming Nie [17] measured normal frost-heave forces of the tunnel and the side of the foundation with vibrating-wire pressure cells, and found out as the temperature varies between 0 and 20 degrees below zero, its frequency has a $5-7 \mathrm{~Hz}$ increase per $10^{\circ} \mathrm{C}$ reduction of temperature and as the temperature varies between 20 degrees below zero and50 degrees below zero, its frequency has a $9-10 \mathrm{~Hz}$ increase per $10^{\circ} \mathrm{C}$ reduction of temperature. Xingui Zhang et al.[18] measured its reading-time curves of randomly selected three earth pressure cells (filled with mercury) under non load and relative temperature-time curves, and found out the trend of readings is in good agreement with that of temperature changing over time, indicating that there exists a linear relationship between the readings and temperature. Yongmei Zhao et al. [19] also confirmed similar linear relationship exists.

Soil water. If the earth pressure cell has waterproof problems, soil water into it may cause an electrical short, or gradually corrodes it, which seriously affect the accuracy of the measured value.

\section{Buried Conditions}

Buried methods. Buried method is correct or not directly related to success or failure of the test. Z. Zhang et al. [20] used two groups of earth pressure cells to measure soil presure in the project of Zhijiang River Reservoir, one group cells embedded in pouring concrete blocks, the results of which completely distorted, the other cells buried in notches on the structural surfaces, the data of which and the analysis results are in good agreement.

Buried spacing. In soil test, the closer the distance between the adjacent soil pressure cells, the stronger their mutual interference, and the more inaccurate the measurement data. Generally, stress measurement in the same direction requires the minimum net distance is not less than 3 times the diameter of the earth pressure cell, at this time there is no mutual interference [4].

Buried depth. Trollope D. H. theoretically deduced, when thickness of covering soil is larger than 0.9 times the effective diameter of the compression face, soil arch effect will produce. At this time, the working state of the earth pressure cell is similar to field condition [21]. Yongli Xie et al [10]. conducted sand calibration which showed the existence of arch effect: when sand filling height is small, the curves of sand calibration and oil calibration curves basically coincide, and the measured stress is close to fill sand self-weight stress; and with sand filling height increasing, the curve is concave, and the difference between sand calibration and oil calibration results becomes increasingly obvious, and the measured stress is much less than the self-weight stress of the filling .

Horizontal position. Earth pressure cells should be calibrated in the same and central location of the test equipment. The experiments showed that the turning points of the curves are different because of the horizontal position of the earth pressure cell in the sand box; the closer to the side wall, the stronger "barn effect", the more significant the difference is, and the calibration test will lose its meaning. In order to further show that the barn effect does exist, the calibration was carried out after vibrating sand box, and the results showed that the turning point of the curve has increased, and "barn effect " gets partially eliminated [10].

Obliquity. Compressed face of the earth pressure cell should be buried perpendicular to the direction of the stress to measure, to guarantee the stability of the measured value. Yankun Li et al.[22] 
figured out using sand calibiration the measured results of cells buried inclined presents poorer linearity than that of ones buried forward, lateral, and reverse.

\section{Loading Method}

Loading history. Loading histories affect the mechanical response of the earth pressure cell [14]. Continuous loading and unloading leads to the continuous reorganization and compression of the particles, pore space shrinking, and the accumulation of particles not be recovered to the initial position and irreversible deformation is increasingly larger, but the increment is smaller and smaller. In addition, with the increase of the load, the decrease of the pore space between the particles leads to the restriction of the sliding recombination space, the energy dissipation decreases, the area of the closed area decreases, and the corresponding strain increases gradually.

Load transfer path. Diffusion leads to the load applied to the cell is not the designed one, and whether the load is applied to the cell affect its matching error. Moreover,loading plate disturbances impacts its reading, resulting in reading jumping.

\section{Improvement Measures}

Many theoretical and experimental research has shown that the thickness-to-diameter ratio $\mathrm{H} / \mathrm{D}$ and the ratio Eg/Es between soil media and modulus are the core factors, and the main way to reduce the effect is to choose appropriate earth pressure cell. And other factors mostly can get improved through adjusting test conditions and loading and unloading method. Based on the analysis of the last section and the research results of domestic and foreign scholars, this paper puts forward the following suggestions:

(a) in order to ensure that there is a good linear correlation between the calibration curve and the grain size of the medium, the diameter of the earth pressure cell should not be less than 4 times the maximum size of the medium. Furthermore, in order to effectively reduce the stress concentration phenomenon caused by the embedding of the earth pressure cell, the effective pressure bearing part of it should not be less than 50 times the maximum size of the measured medium.

(b) the ratio $\mathrm{H} / \mathrm{D}$ should not be larger than 0.2 , the suitable compression plate deflection $\delta$ is $\mathrm{D} /(5000-10000)$, the sensitivity should be moderate, it's best to take $4 \%$ as the limit indicator of zero drift, and to reduce the hysteresis, the earth presssure cell should has a high linear elastic limit, or take some measures for hysteresis self-compensation.

(c) in order to avoid the mutual interference between the cells, the adjacent buried minimum net distance of them should not less than 3 times the diameter of the cell. And the cell should be buried just below the load application point, its compression face should be perpendicular to the stress to measure, and the calibration should be done in the same position. In addition, the boundary friction should be reduced as far as possible by taking some measures such as brushing lubricating oil or pasting plastic films on the wall of the calibration tank

(d) take effective measures to weaken the arch effect because of the cell'sembedding, such as casting some medium generallysilty sand aroud the cell, placing an asphalt bag or elastic rubber pad on the cell.

(e) just above the cell, place a ring with a slightly larger diameter than that of the cell, and between the loading plate and the medium place such buffer materials as liquid bag and foam board, to guarantee the calibration load is applied to the cell effectively, to decrease soil arch effect and the effect of disturbance producing at the loading point.

(f) use earth pressure soil with temperature self-compensating or test out temperature-strain (or frequency) curve for post correction before test.

\section{Conclusions}

Earth pressure cell is an important instrument for measuring soil pressure, the calibration of which is related to the accuracy of the measured value in the real work environment, and then affects the engineering practice and the theoretical innovation. Based on the existing research results, this paper 
analyzes various factors that affect the calibration and puts forward some improvement measures, in order to provide the help for the selection and the calibration of the earth pressure cell.

\section{Acknowledgements}

This work was financially supported by the Industry-university-research Cooperation Program of Henan Province Science and Technology Agency (2015HNCXY009).

\section{References}

[1] Chunlin Li, Qingchun Chen and Qishuo Ding: Calibration of soil pressure sensors for soil compaction monitoring. Experimental Technology and Management, Vol.27(2010), n4, p.63-66.

[2] Chunhong Chen, Sujing Liu and Zhao Wang: Calibration of Earth Pressure Cell. China Rural Water and Hydropower, (2007), n2, p.29-32.

[3] Shunyan Zheng: Discussion on the calibration methods of soil pressure cell. Journal of Highway and Transportation Research and Development: Application Technology Edition , (2013), n1, p.236-238.

[4] Sijiang Wei, Dashun Wang and Jinhai Gao, et al.: Calibration and Correction of Miniature Earth Pressure Cell. Chinese Journal of Underground Space and Engineering, Vol.5(2009), n5, p.1003-1006.

[5] Bin Zhang, Zhao Wang and Junfeng Yang, et al.: Error Analysis of Application of Earth Pressure Cell in Geotechnical Engineering. Exploration Engineering(Rock \& Soil Drilling and Tunneling), Vol.32( 2005), z1, p.157-161.

[6] Shenli Zhang: Analysis of Interaction Characteristics between Soil Pressure Sensor and Soil Medium (Southwest Jiaotong University, China 2010)

[7] Lixiang Zhang, Qiang Luo and Liang Zhang, et al.: Analysis of nonlinear response of soil pressure transducer in high-modulus soil. Rock and Soil Mechanics, Vol.34(2013), n12, p.3633-3640.

[8] Baoyou Liu: General situation of foreign theoretical and experimental research on earth pressure sensor. Journal of Transducer Technology, (1988), n2, p48-53+56.

[9] Yuanming Zuo: Calibration of Earth Pressure Cell. Journal of Nanjing Hydraulic Research Institute, (1981), n1, p.3-34.

[10]Yongli Xie, Xiaomou Wang: Calibration and Analysis of Miniature Resistance-Strain -Transforming Pressure Cell. Journal of Chang'an University(Natural Science Edition), Vol.13(1993), n3, p.23-27.

[11] Labuz J. F., Theroux B.: Laboratory calibration of earth pressure cells. Geotechnical Testing Journal, Vol.28(2005), n2, p.188-196.

[12] Diping Hang, Yunshan Han: Calibration of earth pressure cell. Henan Building Materials, (2014), n1, p.110-112.

[13] Yongxing Guo, Dongshen Zhang and Hui Meng, et al.: Influence of Earth Medium on Earth Pressure Sensor Measurement. Chinese Journal of Underground Space and Engineering, Vol.10 (2014), n2, p.369-376+384.

[14] Xianshan Liu, Cunjun Dong: Mechnaical Response Characteristics of the Earth Pressure Cell in Sand. Journal of Civil, Architectural \& Environmental Engineering, Vol.34(2012), n4, p12-18. 
[15] Yabing Qiu, Shen Zhu and Chen Xu: Calibration Test of Earth Pressure Cell Based on Grade Scale of Field Soil. Water Power, Vol.41(2015), n6, p.118-121.

[16] Dalgle L.: Temperature influence on earth pressure cell readings. Geotechnical News, Vol.23 (2005), n4, p.32-36.

[17] Fengming Nie: Application of vibrating-wire pressure cell in frozen soil. Journal of Glaciology and Geocryology, (1981), n2, p.61-62.

[18] Xingui Zhang, Nianping Yi and Shanshan Wei, et al.: Test method for eliminating the influence of temperature stress on soil pressure cell. Industrial Construction, (2004),z2, p.349-351.

[19] Yongmei Zhao, Jijun Zhang and Guofeng Pan, et al.: Design of tempreature self-compensation fober Bragg grating soil pressure sensor. Transducer and Microsystem Technologies, Vol.33 (2014), n12, p.76-78+81.

[20] Z. Wang, J. Zhang and B. Zhang, et a1.: Two cases histories of application of earth pressure cell. ( Proc. Sino-Japanese symposium on geotechnical engineering, Beijing, China 2003).

[21] Baoyou Liu: Calibration method of earth pressure sensor. Journal of Transducer Technology, (1990), n3, p42-45+63.

[22] Yankun Li, Ke Wang, Yuewei Hao: Experimental study on calibration of different embedding conditions. Geotechnical Engineering World, Vol.11(2008), n11, p.67-69. 